\title{
La inclusion social en el Perú a través de las bibliotecas püblicas
}

\author{
Fortunato CONTRERAS CONTRERAS \\ universidad Nacional Mayor de San Marcos \\ $\&$
}

Resumen

Se describe brevemente el concepto de exclusión social presentando algunos indicadores de la pobreza en el Perú. Se incorpora la tasa de distribución de bibliotecas públicas a nivel nacional como un indicador de exclusión social y la manera como éstas pueden convertirse en espacios sociales para la inclusión social.

Palabras claves: Bibliotecas páblicas, exclusión social, inclusión social.

\begin{abstract}
Abstact
Concepts such as exclusion and deprivation are here introduced to explain the degrees of poverty in Perú. Shortage of public libraries being is a matter of exclusiveness, this article offers hints on how they might tum into social paces of inclusiveness.
\end{abstract}

Key words: Prblic libraries, social exclusion, social inclusion.

\section{Introducción}

7 dojetivo del presente artículo no es describir las características de Hla exclusión social y de la pobreza, ni realizar estimaciones cuantitativas. Existen muchos estudios en ese sentido; más bien el trabajo pretende inconporar una variable, el de las bibliotecas públicas, que permita identificar cómo se da la exclusión a través de ellas y la manera cómo pueden ser elementos que permitirían la inclusión social.

Inicialmente el concepto de exclusión social se utilizó en Europa (primero en Francia, Italia y los países nórdicos) para hacer referencia a los nuevos problemas sociales y económicos asociados a la globalización como el empleo precario y el subempleo, la inserción 
económica, política y cultural de los inmigrantes o la desintegración social producto de diferencias étnicas. Específicamente, se definía exclusión social como los mecanismos a través de los cuales personas y grupos eran despojados de la participación y titularidad de los derechos sociales, o como un proceso que excluía a una parte de la población del disfnute de las oportunidades económicas y sociales. En ese contexto, la noción de exclusión social se presentaba más amplia que el concepto de pobreza ya que representaba un fenómeno que correspondía a la exclusión de los mercados, pero también de las instituciones sociales y arlturales.

En América Latina, el concepto de exclusión ha sido utilizado con algunas variaciones en diferentes contextos para explicar los fenómenos de marginalidad y pobreza que se observan en el continente. La noción de exclusión social hace referencia a un conjunto de procesos que contribuyen a aumentar la vulnerabilidad de ciertos grupos sociales frente a factores de riesgo que pueden llevarlos a una situación de pobreza extrema. La exclusión social se puede definir como la imposibilidad de un sujeto o grupo social para participar efectivamente a nivel económico, social, alltural, político e institucional. El concepto de exclusión social incluye al menos tres dimensiones: (i) económica, en términos de privación material y acceso a mercados y servicios que garanticen las necesidades básicas; (ii) política e institucional, en aranto a carencia de derechos civiles y políticas que garanticen la participación ciudadana y; (iii) sociocultural, referida al desconocimiento de las identidades y particularidades de género, generacionales, étnicas, religiosas o las preferencias o tendencias de ciertos individuas y grupos sociales. En este contexto, la exclusión social se puede representar como la acumulación en el tiempo y el espacio de riesgos específicos que dificultan o impiden la realización de ciertos derechos (civiles, económicos, sociales, allturales y políticas) y la integración del grupo social afectado con su medio o la sociedad.

\section{Conceptos iniciales básicos}

La realidad social cambia, evoluciona con el tiempo. Los conceptos que la describen modifican a menudo sus contenidos, no son categorías eternas. Intentar captar la esencia de un fenómeno social exige no 
anclarse en concepciones que puedan estar desfasadas, que no expliquen correctamente la realidad, porque errar en los diagnósticos puede generar que los esfuerzos por transformarla resulten estériles, ineficientes o que, incluso, produzcan efectos contraproducentes. Continuamente se producen nuevas formas de pobreza y de exclusión social, surgen nuevos grupos de riesgo, se modifican las zonas de vulnerabilidad, por lo que los conceptos y fórmulas de medición deben poder adaptarse a esa realidad cambiante.

El concepto de pobreza se suele caracterizar por la ausencia de recursos mínimos suficientes y de ciertas capacidades para atender las necesidades básicas de personas, familias o colectivos, por lo tanto, hace referencia a aspectos de desigualdad económica y a situaciones de dependencia. Pero la noción de pobreza resulta insuficiente para entender adecuadamente muchos de los fenómenos sociales que se procucen en la realidad y que no pueden ser explicados simplemente en términos de carencias de recursos económicos y de capacidades. Por ello se ha acuñado un concepto mucho más global y flexible, que es el de la exclusión social, con el objeto de intentar enfocar unos procesos que van más allá de la mera desigualdad social y que poseen múltiples dimensiones.

«El concepto de exclusión social se refiere a la acción y efecto de impedir la participación de ciertos grupos sociales en aspectos considerados como valiosos de la vida colectiva. La exclusión social es el reverso de la integración social» ${ }^{1}$, se considera a la exclusión social de manera amplia como la imposibilidad de una persona o de un grupo social para participar activamente en las esferas económicas, culturales, políticas, educativas, culturales o institucionales de la sociedad. Ia exclusión social es una condición tanto objetiva como subjetiva que puede cambiar en el tiempo y el espacio en la medida en que la forman procesos históricos y sociales específicos. Ia exclusión social hace referencia, pues, a los procesos, las situaciones y los mecanismos en función de los cuales una parte de la población (personas, grupos o territorios) quedan limitados o ajenos a la participación en los intercambios, prácticas y derechos sociales y económicos reconocidos en el marco de un Estado Social y de Derecho. 
El término pobreza tiene relación con la distribución del ingreso y su apropiación desigual por parte de individuas y grupos sociales diferentes, que origina distintas capacidades de consumo entre los segmentos extremos de la población, por lo que se trata de un análisis específico de la desigualdad económica. En este sentido, se define un determinado nivel de recursos -umbral de la pobrezade forma que para el segmento situado por debajo de esta línea no es posible alcanzar el nivel de vida considerado la norma mínima en una sociedad y en una época determinadas. La exclusión social tiene que ver con las relaciones sociales de producción y su análisis debe diferenciarse del de la pobreza, pues se trata de procesos que impiden a determinados individuos o grupos alcanzar una posición de autonomía que les permita acceder, de forma efectiva, a sus derechos fundamentales (educación, trabajo, vivienda, asistencia sanitaria, altura, etc.) .

\section{Breves datos de la exclusión social en el Perú}

En el último decenio, es decir entre 1993 y 2003 la población peruana ha experimentado un crecimiento del 18\%, lo que en cifras absolutas representa un aumento de 4.1 millones, es decir la población a aumentado de 23.0 millones de habitantes en el año 1993 a 27.1 en el año 2003. Este incremento de la población ha ido acompañado de una caída en las tasas de crecimiento que van de 1.9 para el quinquenio 1990-95 a 1,7 para 1995-2000 y de 1,5 para el periodo comprendido entre 2001 y el 2003.

En cuanto a la estructura de la población por edades, se doserva una disminución del grupo de 0 a 16 años, el cual de ser mayoritario en 1993 con una participación del 41,6 \%, pasa a ocupar el segundo lugar en el año 2003 con un 37,4 \%, después del grupo de 25 a 44 años de edad que representa un 38, 3\% de la población, según se muestra en el cuadro $N^{\circ} 1$. 
Cuadro $\mathbf{N}^{\circ}$ 1. Perú: estimaciones y proyecciones de la población de ambos sexos por años calendario y edades simples.

\begin{tabular}{|c|c|c|c|c|c|c|c|c|}
\hline \multirow{2}{*}{$\begin{array}{l}\text { Grupos } \\
\text { etáreos }\end{array}$} & \multicolumn{2}{|l|}{1993} & \multicolumn{2}{|c|}{2000} & \multicolumn{2}{|l|}{2002} & \multicolumn{2}{|l|}{2003} \\
\hline & V. Abs. & $\%$ & V. Abs. & $\%$ & V. Abs. & $\%$ & V. Abs. & $\%$ \\
\hline Total & 23009482 & 100,0 & 25939329 & 100,0 & 26748976 & 100,0 & 27148099 & 100,0 \\
\hline $0-5$ & 3637990 & 15,9 & 3698479 & 14,2 & 3664001 & 13,7 & 3640582 & 13,5 \\
\hline 6-11 & 3325142 & 14,5 & 3576507 & 13,8 & 3612451 & 13,6 & 3621894 & 13,3 \\
\hline $12-16$ & 2599149 & 11,2 & 2761233 & 10,6 & 2831978 & 10,5 & 2870565 & 10,6 \\
\hline $17-24$ & 3656480 & 15,9 & 4067639 & 15,6 & 4142459 & 15,4 & 4173798 & 15,3 \\
\hline $25-34$ & 3553828 & 15,4 & 4102660 & 15,9 & 4283617 & 16 & 4377676 & 16,1 \\
\hline $35-44$ & 2474937 & 10,8 & 3065330 & 11,9 & 3237003 & 12,1 & 3322489 & 12,2 \\
\hline 45-más & 3761956 & 16,3 & 4667481 & 18 & 4977467 & 18,7 & 5141095 & 19 \\
\hline
\end{tabular}

Fuente: CEPAL / CEIADE - División de Población. Boletín Demográfico Nº 66 de julio de 2000.

El Perú es uno de los países con el mayor grado de desigualdad y exclusión social, económica, educativa, cultural, etc. Existen indicadores para medir la exclusión. Mencionemos los siguientes:

Distribución del ingreso

Pobreza

PEA ocupada y desocupada

Gastos del gobiemo en educación, salud, vivienda y empleo

Acceso a la seguridad social

Analfabetismo

Indice de Desarrollo Humano

Otro indicador es que el Perú tiene una de las economías más pobres de la región, con un Producto Bnuto Per Capita que presenta una tendencia de crecimiento lenta, produciéndose pocas variaciones con respecto a las cifras del Producto Bruto Intemo, lo que se puede observar en el cuadro $\mathrm{N}^{\circ} 2$. 
Cuadro No 2. Perú-producto bruto interno per cápita.

\begin{tabular}{|c|c|c|c|c|c|c|}
\hline \multirow[t]{2}{*}{ Año } & \multirow[b]{2}{*}{$\begin{array}{c}\text { सBI (1) } \\
\text { (Mi Ilones de } \\
\text { nuevos soles } \\
\text { a precios de } \\
\text { 1994) }\end{array}$} & \multirow{2}{*}{$\begin{array}{c}\text { Población (2) } \\
\text { (Miles) }\end{array}$} & \multirow{2}{*}{$\begin{array}{l}\text { PBI Per capita (3) } \\
\text { (soles a precios } \\
\text { de 1994) }\end{array}$} & \multicolumn{3}{|c|}{ Variación porcentual } \\
\hline & & & & (1) & (2) & (3) \\
\hline 1990 & 81.983 & $21.569,3$ & $3.800,9$ & $-5,1$ & 1,9 & $-6,9$ \\
\hline 1991 & 83.765 & $21.966,4$ & $3.813,3$ & 2,2 & 1,8 & 0,3 \\
\hline 1992 & 83.401 & $22.354,4$ & $3.730,9$ & $-0,4$ & 1,8 & $-2,2$ \\
\hline 1993 & 87.375 & $22.740,2$ & $3.842,3$ & 4,8 & 1,7 & 3,0 \\
\hline 1994 & 98.577 & $23.130,3$ & $4.261,8$ & 12,8 & 1,7 & 10,9 \\
\hline 1995 & 107.039 & $23.531,7$ & $4.548,7$ & 8,6 & 1,7 & 6,7 \\
\hline 1996 & 109.709 & $23.946,8$ & $4.581,4$ & 2,5 & 1,8 & 0,7 \\
\hline 1997 & 117.110 & $24.371,0$ & $4.805,3$ & 6,7 & 1,8 & 4,9 \\
\hline 1998 & 116.485 & $24.800,8$ & $4.696,8$ & $-0,5$ & 1,8 & $-2,3$ \\
\hline 1999 & 117.589 & $25.232,2$ & $4.660,3$ & 0,9 & 1,7 & $-0,8$ \\
\hline 2000 & 121.267 & $25.661,7$ & $4.725,6$ & 3,1 & 1,7 & 1,4 \\
\hline 2001 & 121.513 & $26.090,3$ & $4.657,4$ & 0,2 & 1,7 & $-1,4$ \\
\hline
\end{tabular}

Fuente: Instituto Nacional de Estadística e Informática (INEI) y BCR

La pobreza tiene particular incidencia en el desarrollo de las personas reduciendo sus posibilidades de supervivencia, de una vida sana libre del hambre y de las enfermedades, de los temores y de la exclusión social y por lo tanto, es una situación de falta de oportunidades para un desarrollo verdaderamente humano.

El Perú es uno de los países más pobres de la región, siendo uno de los indicadores la situación en que vive la mayoría de la población. Según las estimaciones de la Encuesta Nacional de Hogares realizada por el INEI a fines del año 2001 (ENAHO 2001), en nuestro país existen 14 millones $609 \mathrm{mil}$ personas que viven en situación de pobreza, dentro de las cuales 6 millones 513 mil se encuentran en condiciones de pobreza extrema. La situación es más alarmante en las áreas nurales, donde la pobreza tiene un carácter mas estructural; y donde de los 7 millones $380 \mathrm{mil}$ personas que se encuentran en situación de pobreza, 4 millones viven en pobreza extrema. 
En el área urbana, incluyendo Lima Metropolitana, la población que vive en situación de pobreza llega a 7 millones $280 \mathrm{mil}$, de los cuales 1 millón 715 mil son personas en situación de pobreza extrema. Sus características son un alto índice de trabajo independiente y familiar con escasa productividad, la escasez de posibilidades de generar empleo adecuado y una pequeña proporción de población ocupada en el sector formal. Su nivel de vida es sumamente bajo, con viviendas precarias en situación de hacinamiento y carencia de servicios básicos de agua, desagüe y electricidad.

En las áreas nurales el poder adquisitivo de las familias, medido a través del ingreso anual del hogar per cápita, es sistemáticamente bajo en relación al de sus semejantes en las áreas unbanas, lo cual se puede apreciar en el cuadro $\mathrm{N}^{\circ} 3$.

Cuadro $N^{\circ}$ 3. Ingreso anual del hogar per cápita* según dominios de estudio. (índices, Lima metropolitana $=100$ )

\begin{tabular}{ll}
\hline Dominios de estudio & Indice \\
Lima Metropolitana & 100 \\
Sierra urbana & 85 \\
Costa urbana (no incluye Lima) & 70 \\
Selva urbana & 67 \\
Costa nural & 45 \\
Sienranural & 39 \\
Selvanural & 38 \\
\hline
\end{tabular}

Nota: *A precios de Lima metropolitana de mayo del 2000. En valores monetarios para Lima metropolitana el ingreso anual del hogar per cápita es S/. 5, 620 .

Fuente: Instituto Cuánto-ENNIV 2000.

Al comparar el ingreso anual per cápita promedio de los hogares según área de residencia, con respecto al promedio en los hogares de Lima metropolitana, se observa que los hogares rurales muestran un menor poder adquisitivo, el cual no llega a ser ni el 50\% de aquél en los hogares de Lima metropolitana. 
Tomando los indicadores de la Pobreza, que nos demuestra sus niveles en el país, el INEI realizó en el último trimestre del 2001 la Encuesta de Condiciones de Vida y pobreza ENAHO IV a nivel departamental.

A fines del año 2001 en el Perú existían 14 millones 609 mil personas en situación de pobreza, dentro de las cuales 6 millones 513 mil se sitúan en el nivel de extrema pobreza. En el medio rural, la situación tiende a ser más crítica: La pobreza afecta a 8 de cada 10 peruanos y la pobreza extrema a 5 de cada 10 .

El porcentaje de pobres a nivel nacional alcanzaba el 54.8\% del total de la población en el año 2001. Esto significa que más de la mitad de la población tenía un nivel de gasto insuficiente como para adquirir la canasta básica de consumo. Sin embargo, el promedio nacional oculta situaciones fuertemente contrastadas según áreas y a nivel regional.

La pobreza en el área rural alcanzaba al 78,4\%, mientras que en el área urbana al 42\%. Una tendencia similar se presenta en la pobreza extrema, que es superior en más de cinco veces en el área rural (51,3\%) que en el área urbana (9,9\%). Un Estado tradicionalmente centralista y la persistencia de políticas sociales asistencialistas y verticales, contribuyeron a profundizar esta brecha.

En la costa el porcentaje de pobreza alcanza el 39,3\%, la sierra 72,0\% y la selva 66, 7\% (ver Cuadro $N^{\circ} 4$ ) .

Cuadro $\mathbf{N}^{\circ}$ 4. Incidencia de la pobreza total y extrema pobreza según dominios geograficos en 2001 (fgt0) .

\begin{tabular}{lcc}
\hline Daminios greograficos & Tasa de pobreza total\% & $\begin{array}{c}\text { Tasa de pobreza } \\
\text { extrema\% }\end{array}$ \\
\hline Nécional & 54,8 & 24,4 \\
Áreas & & \\
Urbana & 42,0 & 8,9 \\
Rural & 78,4 & 48,0 \\
Regiones Naturales & & \\
Costa & 39,3 & 5,8 \\
Siena & 72,0 & 45,6 \\
Selva & 66,7 & 39,7 \\
\hline
\end{tabular}

Fuente: INEI. Encuesta de Condiciones de Vida y Pobreza-ENAHO IV. 2001. 
La pobreza a nivel departamental nos muestra los departamentos que tienen los indicadores más altos de pobreza como son: Huancavelica con 88,0\% de pobreza total, Huánuco con 78, 9\%, Puno con 78, 0\%, Apurímac con 78,0\%, Cajamarca con 77,4\%, Cusco con 75,3\%, Amazonas 74,5\%, Ayacucho con 72,5\%, Ucayali con 70,5\%, Loreto con 70, 0\%, San Martín con 66,9\%, y así sucesivamente y los departamentos menos pobres Moquegua con 29,6\%, Tacna con 32, 8\%, Lima con 33,4\%, Madre de Dios con 36,7\%, etc.. (ver Cuadro $\mathrm{N}^{\circ} 5$ ) .

Cuadro $\mathbf{N}^{\circ}$ 5. Incidencia de la pobreza total y extrema pobreza según departamentos 2001 (fgt0).

\begin{tabular}{lcc}
\hline Departamento & Tasa de pobreza total \% & $\begin{array}{c}\text { Tasa de pobreza } \\
\text { extrema\% }\end{array}$ \\
\hline Amazonas & 74,5 & 41,1 \\
Ancash & 61,1 & 33,3 \\
Apurímac & 78,0 & 47,4 \\
Arequipa & 44,1 & 14,5 \\
Ayacucho & 72,5 & 45,4 \\
Cajamarca & 77,4 & 50,8 \\
Cusco & 75,3 & 51,3 \\
Hirancavelica & 88,0 & 74,4 \\
Huánuco & 78,9 & 61,9 \\
Ia & 41,7 & 8,6 \\
Jinín & 57,5 & 24,3 \\
LaIibertad & 52,1 & 18,3 \\
Lambayeque & 63,0 & 19,9 \\
Lima & 33,4 & 3,1 \\
Loreto & 70,0 & 47,2 \\
Madre De Dios & 36,7 & 11,5 \\
Moquegua & 29,6 & 7,6 \\
Pasco & 66,1 & 33,2 \\
Piura & 63,3 & 21,4 \\
Puno & 78,0 & 46,1 \\
San Martín & 66,9 & 36,2 \\
Tacna & 32,8 & 5,2 \\
Tumbes & 46,8 & 7,4 \\
Ucayali & 70,5 & 44,9 \\
\hline & 6 . &
\end{tabular}

Fuente: INEI. Encuesta de Condiciones de Vida y Pobreza-ENAHO IV. 2001. 


\section{Indicadores de bibliotecas públicas}

Tomando la variable de bibliotecas públicas, tenemos indicadores de acuerdo al registro que tiene la Biblioteca Nacional. En el 2000, en el Penú existía un total de 1576 (100\%) bibliotecas públicas. El número existente a nivel nacional no reflejaba el nivel de desarrollo de las bibliotecas, vale decir no había ninguna institución, incluida la Biblioteca Nacional, que pudiera acreditar el nivel de desarrollo de estas bibliotecas. La verdad es que en este registro hay «bibliotecas públicas» que tienen pocos títulos, infraestructura inadecuada, sin personal profesional o técnico calificado, sin sistematización de la colección bibliográfica, etc. Sin embargo vamos a usar estos indicadores que nos permitirán identificar el grado de exclusión en la distribución de bibliotecas públicas a nivel nacional.

Los departamentos que concentran mayor número de bibliotecas públicas son: Lima con 198 (12,56\%) , Junín con 138 (8,75\%), Puno con $128(8,12 \%)$, Cajamarca con 111 (7,04\%), Ancash con 108 (6,85\%) y así sucesivamente.

Los departamentos que concentran menor número de bibliotecas públicas son: Madre de Dios con 6 (0,38\%), Ucayali, Tacna, Moquegua, todas con $12(0,76 \%)$, y así sucesivamente (ver cuadro $\left.N^{\circ} 6\right)$.

Considerando que las bibliotecas públicas son organizaciones que van experimentando cambios como consecuencia de factores derivados del desarrollo de la sociedad: factores económicos, políticos, sociales, cambios en la educación, aultura, ciencia y de la tecnología, etc. , en el Penú es comḿn escuchar acerca del divorcio de las bibliotecas públicas y la comunidad hacia las cuales deben estar orientadas. Algunos especialistas afirmaron que la sociedad de la información sería una sociedad de inclusión social, en la que los recursos se compartirían, dedicada al bienestar social y en la que la tendencia dominante sería acabar con las desigualdades sociales. No dostante, al iniciarse el nuevo siglo, el discurso ha seguido un camino totalmente contrario. Gran parte de los especialistas de la sociedad de la información coincide que esta es una sociedad perversa, una sociedad feudal donde los que concentran los recursos son los que tienen el poder y acentúan el proceso de desigualdad. 
Cuadro $\mathbf{N}^{\circ}$ 6. Distribucion geografica de las bibliotecas públicas municipales 2000.

\begin{tabular}{|c|c|c|c|c|c|c|c|}
\hline \multicolumn{8}{|c|}{ Bibliotecas Públicas Municipales } \\
\hline \multicolumn{8}{|l|}{$\mathbf{N}^{\circ}$} \\
\hline ORDEN & Departamentos & Provincias & Distritos & C.P.M & Ag. Mrm. & Total & $\%$ \\
\hline 1 & Amazonas & 7 & 43 & 9 & 3 & 62 & 3.93 \\
\hline 2 & Ancash & 20 & 80 & 2 & 6 & 108 & 6.85 \\
\hline 3 & Apurímac & 7 & 41 & 2 & 4 & 54 & 3.42 \\
\hline 4 & Arequipa & 8 & 27 & & 1 & 36 & 2.28 \\
\hline 5 & Ayacucho & 11 & 67 & 1 & 9 & 88 & 5.58 \\
\hline 6 & Cajamarca & 13 & 66 & 9 & 23 & 111 & 7.04 \\
\hline 7 & Cusco & 13 & 59 & 1 & 5 & 78 & 4.94 \\
\hline 8 & Huancavelica & 7 & 64 & 19 & 17 & 107 & 6.78 \\
\hline 9 & Huánuco & 10 & 49 & 1 & 8 & 68 & 4.31 \\
\hline 10 & $\mathrm{Ia}$ & 5 & 28 & & 1 & 34 & 2.15 \\
\hline 11 & Junín & 9 & 100 & 1 & 28 & 138 & 8.75 \\
\hline 12 & La Libertad & 11 & 50 & 5 & 6 & 72 & 1.58 \\
\hline 13 & Lambayeque & 3 & 25 & & 2 & 30 & 1.90 \\
\hline 14 & Lima & 11 & 132 & 2 & 53 & 198 & 12.56 \\
\hline 15 & Loreto & 5 & 17 & & 3 & 25 & 1.58 \\
\hline 16 & Madre de Dios & 3 & 3 & & & 6 & 0.38 \\
\hline 17 & Moquegua & 3 & 8 & & 1 & 12 & 0.76 \\
\hline 18 & Pasco & 3 & 17 & 11 & 8 & 39 & 2.47 \\
\hline 19 & Pinra & 8 & 50 & 5 & 23 & 86 & 5.45 \\
\hline 20 & Puno & 13 & 86 & 12 & 17 & 128 & 8.12 \\
\hline 21 & San Martín & 9 & 40 & 3 & 4 & 56 & 3.55 \\
\hline 22 & Tacna & 4 & 8 & & & 12 & 0.76 \\
\hline 23 & Tumbes & 3 & & 12 & 1 & 16 & 1.01 \\
\hline \multirow[t]{3}{*}{24} & Ucayali & 4 & & 8 & & 12 & 0.76 \\
\hline & TOTAL & 190 & 1080 & 83 & 223 & 1576 & 100.0 \\
\hline & $\%$ & $12.10 \%$ & $68.50 \%$ & $5.30 \%$ & $14.10 \%$ & $100 \%$ & \\
\hline
\end{tabular}

Fuente: Biblioteca Nacional del Perú. Base de datos del SNB.

El acceso democrático a la información a pesar de que es un derecho de la sociedad peruana, se restringe más a una pequeña parte elitista de la población. La porción restante queda marginada del proceso de ciudadanía, desconociendo los derechos y deberes que les corresponden en la sociedad. Este es el gran conglomerado de los excluidos. Generalmente las personas que conforman los grupos son de bajos niveles económicos, educativos, sociales, etc., y no están documentados. A estos se les denomina los INFOEXCLUIDOS. De este 
grupo surgen generalmente los grandes problemas sociales como el desempleo, la violencia, la desnutrición, la mortalidad, bajos niveles educativos, etc. razón por la cual aumenta cada vez más el porcentaje de la población excluida del proceso social, educativo y cultural.

La desinformación afecta esencialmente a las clases menos favorecidas e influye negativamente en el proceso de desarrollo de la ciudadanía, La desinformación puede significar la falta de información, la información incorrecta, la manipulación de la información. Toob esto lleva a la formación de una sociedad incapaz de discemir, criticar y, consecuentemente, tomar decisiones favorables para su propio desarrollo. Siendo así, es fácil constatar que la exclusión social es también un proceso de desinformación.

\section{Bibliotecas públicas: Espacio de inclusión social}

El Perú ha sido y sigue siendo un país con importantes recursos humanos, naturales, físico-geográficos y culturales, capaces de generar sólidos procesos de desarrollo. La carencia, inestabilidad, debilidad o contradicción de las políticas aplicadas o las propuestas de desarrollo emprendidas en el pasado no han permitido potenciarlos ni aprovecharlos convenientemente.

La reciente recuperación de la democracia abre un nuevo escenario donde las expectativas insatisfechas, las necesidades apremiantes de grandes sectores en el país y las propuestas de cambio, generan una energía social que debe ser encauzada en un marco coherente de acción, que promueva la creatividad colectiva, fortalezca las organizaciones y vele por la acertada orientación de las políticas macro económicas orientadas al desarrollo humano. También el uso de las potencialidades, entendidas como el conjunto de recursos naturales, físicos, humanos, institucionales y económicos existentes que son mal aprovechados o que están ocultos. Usar al máximo posible lo que tenemos o podríamos tener, es la tarea inicial para movilizar las fuerzas locales, regionales y nacionales rumbo al desarrollo humano. El enfoque de las potencialidades fomenta la adopción de una actitud positiva de enfrentar las dificultades que pudiera presentamos la realidad, para sal ir adelante, organizarse y trabajar para el bien común. Esta actitud 
debe abrir nuevas oportunidades dentro de esquemas económicos y sociales que minimicen las desigualdades extremas entre las personas y agrupaciones para acceder a la modernidad, garantizándoles ingresos y otros bienes intangibles que los coloca dentro del paradigma del Desarrollo Humano. Estos elementos elevan la autoestima colectiva y fortalecen la sensación de pertenencia a un colectivo social.

El énfasis de este enfoque radica en el conocimiento y utilización de muestras potencialidades, para sobre esa base desencadenar procesos que impliquen el logro de objetivos vinculados al bienestar, al crecimiento de la economía y a la equidad distributiva entre los diferentes sectores sociales. El punto es, entonces, buscar las combinaciones adecuadas para dar valor presente a nuestras disponibilidades, en lugar de sólo insistir sobre las demandas de lo que nos falta.

Según el paradigma del desarrollo humano, que propugna el Programa de las Naciones Unidas para el Desarrollo (PNUD), la meta del desarrollo no se expresa exclusivamente por el crecimiento económico. Este es un medio no el fin del desarrollo. Ell fin del desarrollo tiene que ver con la existencia de oportunidades para todos: que la gente viva más años, con mejor calidad de vida, que tenga libertades, acceso a la educación, a la cultura y a otras áreas que le permitan su plena realización personal.

Por otro lado, el desarrollo humano debe ser sostenible. Tanto en lo relacionado a la conservación de los recursos naturales para las siguientes generaciones; como asegurar que los logros del desarrollo actual se consoliden para evitar retrocesos.

Las bibliotecas públicas conceptualmente están concebidas para servir a las sociedades a través de la democratización de la información, el ocio, el acceso al conocimiento, la cultura, etc. Ia biblioteca es un espacio dinamizador de la sociedad y de promoción de la igualdad de oportunidades.

Las bibliotecas públicas son espacios de carácter cívico, que nos pertenecen a todos y a nadie en particular. Forman parte de la red de asociaciones que conforman la sociedad civil, muchas de las cuales son de naturaleza informal, y que contribuyen a generar capital social y acogen a todo el mundo. La biblioteca pública no es ya un mero 
depósito pasivo de libros e información, ni un puesto de avanzada cultural, sino una parte activa y sensible de la commidad y un agente de cambio. Las bibliotecas públicas tienen a su disposición nuevos modos de relacionar a los vecinos, reavivar el compromiso cívico, impulsar la participación ciudadana y fomentar un mayor grado de intervención en los procesos de decisión y solución de los problemas de la comunidad.

Parece, pues, evidente que las bibliotecas continúan encontrando su razón de ser en el desarrollo y profundización de la sociedad, en el ejercicio del derecho a la información y la garantía de la igualdad de oportunidades. Proporcionar un acceso libre y equitativo a información de calidad, que reduzca la disparidad entre ciudadanos ricos y ciudadanos pobres en información, adquiere actualmente una especial relevancia para amplios sectores de la población.

Las bibliotecas son espacios sociales que encaman la educación, la cultura y otros importantes valores seculares y manifestaciones del bien común, y la necesidad de este tipo de espacios nace de la propia naturaleza humana. La biblioteca pública es un patrimonio de la comunidad a la que sirve, pues apoya y estimula la formación permanente, además de proporcionar un acceso igualitario a la información. Aparte de su función vital de atención a las personas, la biblioteca es un instrumento de su comunidad. La biblioteca pública es un recurso para atender a las prioridades de la commidad, como el desarrollo econámico, la revitalización de los barrios, la participación ciudadana, la formación permanente y la integración

Las directrices para el desarrollo públicadas por IFLA/UNESCO describen el importante papel que desempeña la biblioteca pública como espacio público y punto de encuentro. El capital social son las redes, las normas y la confianza social que facilitan la coordinación y la cooperación en beneficio mutuo. La biblioteca crea capital social y fomenta el compromiso cívico promoviendo las asociaciones comunitarias, facilitando el diálogo local y divulgando información local. Existen estudios que demuestran que un mayor capital social estimula una mayor productividad, rebaja las tasas de enfermedad, reduce la delincuencia, aumenta las puntuaciones obtenidas en las pruebas e inspira mayor sensibilidad a la acción de gobiemo. Si la 
biblioteca se orienta a estas actividades de creación de capital social, cumplirá con reavivar el compromiso cívico, impulsar la participación ciudadana y fomentar un mayor grado de intervención en los procesos de decisión y solución de los problemas de la comunidad.

En estrecha colaboración con todos los sectores de la ciudadanía, la biblioteca puede ayudar a su commidad a reavivar la sociedad civil y aumentar la participación ciudadana. Sin la sociedad civil y las relaciones sociales, los ciudadanos se quedan inermes ante los gobiernos burocráticos y los mercados privados. Para las comunidades resulta imprescindible disponer de un espacio público y comunitario. La biblioteca pública está abierta a toobs los que llegan a ella, ofreciénobles igualdad de oportunidades de acceso y participación en la sociedad.

\section{Acciones a tomar}

Para reducir la desigualdad y por lo tanto la exclusión social a través de las bibliotecas públicas en pos de la integración de la sociedad, la igualdad de oportunidades y la generación de capacidades se recomienda:

A nivel de la sociedad y del Estado:

1) La participación del Estadb a través de las políticas sociales mediante la creación, promoción y financiamiento de las bibliotecas públicas que permitan la redistribución en el acceso de la información.

2) La participación de la sociedad civil en la promoción, mantenimiento y vigilancia ciudadana de las bibliotecas públicas.

3) La concentización de la importancia de las bibliotecas públicas y participación de los gobiemos regionales en la creación, promoción y financiamiento de las bibliotecas públicas a nivel regional.

A nivel de instituciones de académicas, técnicas y normativas:

1) Acreditar a las bibliotecas públicas según niveles de desarrollo siguiendo el modelo de acreditación de los hospitales, hoteles, restaurantes, etc.

2) Elaborar un índice de desarrollo de las bibliotecas püblicas, algo así como el índice de desarrollo humano o índice de la pobreza.

3) Ellaborar estadísticas más detalladas sobre las bibliotecas públicas que inconporen: múmero de usuarios reales y potenciales, personal 
profesional, técnico, infraestnuctura, número de títulos de material bibliográfico, mobiliario, equipos, normas de sistematización, demanda y necesidades de información, indicadores de inversión en las bibliotecas públicas, etc.

4) Elaborar diagnósticos participativos de las bibliotecas públicas a nivel departamental.

5) Elaborar indicadores que permitan medir cómo las bibliotecas públicas están contribuyendo al desarrollo de la comunidad; vale decir, qué cambios están sucediendo en la commidad a partir de la intervención de la biblioteca pública.

A nivel departamental:

1) Inconporar en las mesas de concertación social y de lucha contra la pabreza, el tema de las bibliotecas públicas.

2) La participación real del sistema nacional de bibliotecas a nivel nacional en su rol técnico normativo.

3) La participación de las ONGs en la creación, promoción y financiamiento de las bibliotecas públicas.

4) Elaborar investigaciones sobre la relación entre las bibliotecas públicas y temas sociales, políticos, culturales, educativos, participación ciudadana, relaciones biblioteca-commidad, exclusión social, servicios de información local, commitarios e información y democracia, entre otros.

\section{Nota}

1 Figueroa, Adolfo; Altamirano, Teófilo; Sulmont, Denis. Exclusión social y desigualdad en el Perú. OIT, Lima, 1996, p. 19.

\section{Referencias bibliográficas}

BETAR, Héctor (2001) : Justicia social, política social. CHDEP, Lima. CASTELLS, Manuel (1999) : La era de la información. Alianza Editorial, Madrid.

COMISIÓN DE TRANSFERENCIA DE LA BIBLIOTECA NACIONAL DEL PERÚ (2001) : Informe situacional. Documento. Lima. 
FIGUEROA, Adolfo (1996) : Exclusión social y desigualdad en el Peñ́. OIT, Lima.

FIGUEROA, Adblfo (2001) : Reformas en sociedades desiguales: la experiencia penuana. PUCP, Lima.

FRANCKE, PEDRO (2001) : Políticas sociales: balance y propuestas. Lima. FIAB/UNESCO (2001) : Directrices IFLA/UNESCO para el desarrollo del servicio de biblioteca públicas. URL: Consultado el 13/05/2003. http:// unesdoc.unesco.org/images/ 0012/001246/124654s/pdf HERRERA, Javier (2002) : La pobreza en el Perú: una visión departamental. INEE, Lima.

INFOLAC (1999) : Declaración de Caracas sobre la biblioteca pública. URL: Consultado el 13/05/2003. http://infolac.ucol.mx/documentos/ bibliotecas/prin2.html

MACHUCA, Raúl Mauro (2002) : Cambios de la pobreza en el Perń: 19911998. Un análisis a partir de los componentes del ingreso. Lima.

PARODI TRECE, Carlos (2002): EConomía de las políticas sociales.Universidad del Pacifico, Lima.

PASCÓ-FONT QUEVEDO, Alberto (2001) . Reformas estructurales y bienestar: una mirada al Perú de los noventa. GRADE, Lima.

PERÚ. BIBLTOIECA NACIONAL (2002) : La Biblioteca Nacional del Perú: balance y propuestas. BNP, Lima.

SUADEN, Emir José (1999) : «La biblioteca pública y la sociedad de la información : gldbalización y escenarios». En: Ell libro en América Latina yel Caribe, 87 ene./ jun. ; pp. 28-38.

SUAIDEN, Emir José (2002) : «El impacto social de las bibliotecas públicas». En: Anales de Documentación, 5; pp. 333-344.

UNESCO (1994) : Manifiesto de la Unesco sobre la biblioteca pública. URL: Consultado el 13/05/2003. http: ://www.ifla.org/s8/unesco/spain.htm 